\title{
Bicondylar Tibial Plateau Fractures Involving the Posterior Fragment: Injury Mechanism and Fracture Characteristics
}

\section{Shuo Pan}

Hebei Medical University Third Affiliated Hospital

Aqin Peng ( $\sim$ pengaqin@126.com )

Third Hospital of Hebei Medical University https://orcid.org/0000-0003-4449-8210

\section{Yaning $\mathrm{Hu}$}

Hebei Medical University Third Affiliated Hospital

\section{Shuai Wang}

Hebei Medical University Third Affiliated Hospital

\section{Xiao Zhang}

Hebei Medical University Third Affiliated Hospital

\section{Research article}

Keywords: Bicondylar tibial plateau fracture, Injury mechanism, Fracture characteristic, Computer-assisted three-dimensional technique.

Posted Date: March 9th, 2020

DOI: https://doi.org/10.21203/rs.3.rs-16383/v1

License: (c) (1) This work is licensed under a Creative Commons Attribution 4.0 International License. Read Full License 


\section{Abstract}

Background: Bicondylar tibial plateau fracture (BTPF) involving the posterior fragment is the most complex intra-articular fracture to treat. Although the fracture characteristics of BTPFs have been reported by many researchers, due to the sophisticated kinematics of the knee, reports focused on the injury mechanism are still scarce. The current injury mechanism is insufficient to explain the various fracture characteristics and to guide surgery. This study used a three-dimensional (3D) simulation method to investigate the injury mechanism and fracture characteristics of BTPFs involving the posterior fragment. Methods: Ninety-six BTPFs involving the posterior fragment were included. A computer-assisted 3D technique, which enabled us to move the femoral condyles to match the displaced fracture fragments of the tibial plateau, was applied to analysis the injury mechanism for all cases. The 3D and two-dimensional (2D) morphology of each fracture were reviewed thoroughly, and the main fracture lines were mapped and superimposed on a template. Results: After simulation and quantitatively analysis, three main types (extension, flexion-internal rotation, flexion-external rotation) and six sub-types (valgus and varus in each main type) of injury mechanism had been classified according to the degree of knee motion in three planes. In the extension type, femoral condyles compress the anterolateral tibial plateau and central medial plateau, resulting in an anterior-to-posterior fracture line. In the flexion-internal rotation type, the compressed areas are located on the posterolateral and anteromedial plateau, forming an oblique anteromedial-to-posterolateral fracture line. In the flexion-external rotation type, the compressed areas are located on the anterolateral and posteromedial plateau, exhibiting an anterolateral-to-posteromedial fracture line. Conclusions: Different injury mechanisms result in different fracture characteristics. A thorough understanding of the injury mechanisms underlying complex BTPFs involving the posterior fragment is meaningful not only for fracture characteristics reorganization but also for surgical decision making. The 3D simulation method employed in this study may be a useful supplemental method for investigating the mechanisms underlying fracture injuries.

\section{Background}

Posterior fragments of the tibial plateau fracture are difficult to detect on plain radiographs and have historically been observed infrequently, but with the development of imaging methods such as computed tomography (CT), posterior fractures of the tibial plateau are increasingly being recognized and noted [13]. Bicondylar tibial plateau fractures (BTPFs), which are classified as Schatzker type $\mathrm{V}$ or VI, are complex articular fractures with multiple fracture lines and fragments, many of which involve posterior fracture fragments [4-8]. Yang [6] reported 93 posterior fractures out of 149 BTPFs, Zhu [7] reported that $44.32 \%$ of BTPFs involved posterolateral fractures, and Barei [4] identified 66 posteromedial fractures among 146 BTPFs. Posterior fracture fragments, especially in complex BTPFs, are difficult to stabilize by conventional surgical approaches and are always associated with an inferior functional outcome for patients [5].

Treatment decision making for complex tibial plateau fractures requires two aspects to be considered: the three-dimensional (3D) characteristics of the fracture and, more importantly, the mechanism of injury. Currently, the injury mechanism of BTPF is believed to consist of extreme axial force load on the articular surface [9], and posterior fractures of the tibial plateau results from force in a position of flexion $[5,7,10]$. 
However, the mechanism of injury is a matter of speculation based on surgeons' experience and biomechanical experiments, which cannot always explain the various fracture characteristics.

Tibial plateau fractures result from the impact of femoral condyles with the knee joint in various positions in conjunction with a certain deforming force. Reconstructing the position of the knee joint at the time of fracture can provide the opportunity to understand the injury mechanism more precisely and reliably. In this study, we used a computer-assisted 3D technique to simulate the knee position during fracture by moving the femoral condyles to match the displaced articular surfaces of the tibial plateau fracture, and then we analysed the motion of the femur. We hypothesized that the simulation could reveal the injury mechanism of BTPF involving the posterior fragment.

\section{Methods}

Subjects

This retrospective study was performed with the approval of the institutional review board (IRB Protocol \#2019-036-1). One hundred and eighty-three patients with BTPFs treated between December 2016 and November 2019 were identified by searching an orthopaedic database maintained at a level-I trauma centre. Fifty-nine bicondylar fractures without posterior involvement were excluded. Twenty-eight additional fractures had insufficient CT record data available in their Digital Imaging and Communications in Medicine (DICOM) files, and those fractures were also excluded. Patients under 16 years old or with pathological fracture, open proximal tibial fracture, previous knee surgery and/or existing knee ligament malfunction, existing knee deformity, insufficient-quality CT images or axial CT images with a slice thickness $>3 \mathrm{~mm}$ were excluded. Ultimately, a total of ninety-six BTPFs with posterior part fractures, classified as Schatzker types $\mathrm{V}$ and $\mathrm{VI}$, were included in this retrospective review.

After the DICOM files of fractures were imported into Mimics software (19.0, Materialise, Leuven, Belgium), 3D models of the tibia and femur were created separately with the "CT Bone Segmentation" command. Then, the tibial and femoral 3D models were adjusted to the fully extended position as the reference position with standard orientations by using the "Pan" and "Rotate" functions as necessary. For comparative analysis, the right knee 3D models were flipped horizontally to obtain standard left knee models (Fig. 1).

Knee position simulation and analysis

To simulate the knee position at the time of fracture, we used the "reposition" function to move the femoral 3D object to ensure that the articular surfaces of the femoral condyles matched the geometric shapes of the displaced articular surfaces in the tibial plateau fracture. The femur and tibial contour lines in the 2D views were also adjusted to achieve optimal matching in all slices (Fig. 2). The final tibial position was determined by consensus between 2 senior orthopaedic surgeons (S.P. and Y.H.) supervised by the corresponding author (A.P.). 
The motion of femur was analysed by the software, and the rotation values in the three planes were recorded and compared. The 3 items in "Rotation" represent the motion of the femur in different planes relative to the standard extension position (reference position) at the time of the fracture injury. A positive value in the sagittal plane represents flexion, and a negative value represents hyperextension. In the axial plane, a positive value represents external rotation, and a negative value represents internal rotation. In the coronal plane, a positive value represents varus, and a negative value represents valgus.

\section{Fracture mapping}

The method of two-dimensional (2D) fracture mapping was first described by Cole and colleagues [11] and then modified by Molenaars et al. [12] for fracture line depiction. In the current study, we used the 3D topview of the fracture instead axial CT slice of the fracture to comprehensively describe the articular fracture lines in fracture mapping.

Top-view images of 3D models of BTPFs were imported into Adobe Illustrator software (2019, Adobe Systems Incorporated, San Jose, CA) on a 2D standard template of an intact left tibial plateau. Specific tibial plateau landmarks such as the medial and lateral tibial plateau, the tibial tubercle, and the fibula were matched to ensure proper rotation and alignment (Fig. 3).

Main fracture lines were drawn on the template by consensus between 2 senior orthopaedic surgeons (S.P. and Y.H.) based on the axial, sagittal, coronal, and 3D model images; disagreements were resolved by discussion with the corresponding author (A.P.). In every case, the main fracture lines are depicted and overlapped, yielding a 2D diagram with variations in density relative to fracture frequency.

Data analysis

All statistical analyses were performed using SPSS software (version 25.0; IBM). The data on age were subjected to one-way analysis of variance (ANOVA), and the data on sex, fracture side, and Schatzker classification were compared using Fisher's exact test. The injury mechanism "Rotation" data from all three planes were compared among the different types and sub-types using the Kruskal-Wallis and Nemenyi tests. A P $<0.05$ was considered statistically significant. The fracture characterization and main fracture lines of each type were descriptive in nature.

\section{Result}

We reviewed ninety-six CT scans of BTPFs involving the posterior part: thirteen were Schatzker type V and eighty-three were Schatzker type VI tibial plateau fractures. The study subjects were composed of seventy male patients and twenty-six female patients, with an average age of forty-four years (range, twenty to sixty-six years).

Injury mechanism classification

This injury mechanism classification was based on the interpretation of the knee position at the time of fracture, the main types were classified by the motion in sagittal (extension or flexion) and axial plane 
(internal or external rotation), and the sub-types were classified by the motion in coronal plane (valgus or varus). Three main types (extension, flexion-internal rotation, flexion-external rotation) and six sub-types (valgus and varus in each main type) of injury mechanisms were found in this series of BTPFs involving the posterior part. The baseline demographics are summarized in Table 1. Distinct plateau fracture impingement areas (Fig. 3) and different fracture characteristics were observed for each injury mechanism type (Fig. 4-6) (Table 2). The knee position motion data from all the three planes were summarized in Table 3.

\section{Extension injury mechanism}

In the extension position, the knee is under a "lock" pattern, with no obvious additional rotation observed between the tibial and femur. When an axial force and a valgus or a varus force act on the knee, the femoral condyles impact the anterior part of lateral plateau and central part of the medial plateau, leading to the formation of the main fracture lines, which were oriented in an anterior-to-posterior pattern, and the main fracture fragment. Under a valgus force, the anterolateral tibial plateau fracture pattern is mainly a compressed fracture and lateral displacement; with load transfer, the fracture segment of the posterolateral plateau is split and displaced backward. Under a varus force, the medial plateau fractures are split, and in severe cases, transverse lines can be found extending in the distal direction from the middle part (Fig. 4).

In this injury mechanism, sixty (62.5\%) BTPFs were found to have occurred in an extended position $\left(-0.23^{\circ}\right.$ $\pm 6.10^{\circ}$ in the sagittal plane) with no obvious rotation $\left(-0.53^{\circ} \pm 2.63^{\circ}\right.$ in the axial plane). Among those BTPFs, forty-four exhibited a valgus (extension-valgus sub-type), and sixteen exhibited a varus (extension-varus sub-type) injury mechanism $\left(-12.17^{\circ} \pm 4.62^{\circ}\right.$ and $12.52^{\circ} \pm 3.63^{\circ}$, respectively, in the coronal plane; $\left.P=0.000\right)$.

Flexion-internal rotation injury mechanism

In this injury mechanism, the knee is in the flexion and internal rotation position, and the femur exhibits external rotation. The lateral femoral condyle rolls back, impinging the posterolateral quarter of tibial plateau, and the medial femoral condyle moves to the anterior quarter of the medial plateau. When an axial force and a valgus or varus force act on the knee, the femoral condyle impacts the posterolateral and anteromedial tibial plateau, forming the main fracture line, oriented from the posterolateral to anteromedial plateau, and forms the main fracture fragment. Under a valgus force, posterolateral fractures of the tibial plateau exhibit a comminuted and compressed pattern, the fracture lines extend vertically downward from the tibia back, and the fracture pattern of anterolateral quarter is split. Under a varus force, the fragment pattern of medial plateau has an inverted-pyramid shape with anteromedial and distal tips; these fractures are always accompanied by a small posterolateral fragment. An oblique fracture line can be found in the lateral view, and the distal displacement is notable (Fig. 5).

Twenty-one BTPFs exhibited flexion and internal rotation $\left(47.10^{\circ} \pm 13.28^{\circ}\right.$ in the sagittal plane and $-22.39^{\circ}$ $\pm 6.38^{\circ}$ in the axial plane). Among those BTPFs, twelve exhibited a valgus (flexion-internal rotation valgus sub-type) injury mechanism, and nine exhibited a varus (flexion-internal rotation varus sub-pattern) injury mechanism $\left(-12.31^{\circ} \pm 2.43^{\circ}\right.$ and $18.76^{\circ} \pm 8.06^{\circ}$, respectively, in the coronal plane; $\left.P=0.000\right)$. 
Flexion-external rotation injury mechanism

The knee is in the flexion and external rotation position, and the femur exhibits internal rotation. The lateral femoral condyle moves forward and impinges the anterolateral quarter of tibial plateau, and the medial femoral condyle moves to the posterior quarter of the medial plateau. When an axial force and a valgus or varus force act on the knee, the femoral condyle impacts the anterolateral and posteromedial tibial plateau, forming the main fracture line, which originates from the anterolateral to posteromedial plateau, and the main fracture fragment. Under a valgus force, the lateral fractures are mainly compression fractures in the anterolateral part, and the fractured segments of the posterolateral plateau are mainly split but not comminuted. Under a varus force, in the medial plateau, there is invariably a fracture line located between the attachment of the posterior cruciate ligament and the collateral medial ligament, and a small posterior medial plateau fracture is always observed (Fig. 6).

Fifteen BTPFs exhibited a flexion and external rotation $\left(-35.70^{\circ} \pm 8.63^{\circ}\right.$ in the sagittal plane and $-15.13^{\circ}$ $\pm 5.14^{\circ}$ in the axial plane) position. Among those BTPFs, twelve exhibited a valgus (flexion-external rotation valgus sub-pattern), and 3 exhibited a varus (flexion-external rotation varus sub-pattern) injury mechanism $\left(16.71 \pm 4.19^{\circ}\right.$ and $-11.6 \pm 3.09^{\circ}$, respectively, in the coronal plane, $\left.\mathrm{P}=0.004\right)$.

\section{Discussion}

BTPF, especially when it involves the posterior part, is believed to result from the impingement of femoral condyle accompany extreme force. The morphology of the fracture is determined by the applied force and the position of the knee at the time of the injury. Given the various fracture characteristics and complexity of the knee, current knowledge of the injury mechanism is insufficient to understand the fractures, decide on a surgical plan, choose an approach and reconstruct the joint. In this study, we simulated the position of the knee at the time of the fracture using a computer-assisted 3D technique that enabled us to move the distal femoral condyles to match the displaced fracture fragments of the tibial plateau. After simulation for ninety-six BTPFs involving the posterior fragment, we analysed the fracture line orientation, fracture morphology and the motion of the knee in three planes. Three main injury mechanism types and six subtypes were identified and found to exhibit distinct fracture characteristics. We believe that these findings are meaningful for a greater understanding of the complex fractures.

The kinematics of the knee are complex and sophisticated [13, 14]; with different knee positions, the femoral condyles impinge different areas of the tibial plateau, resulting in different fracture characteristics (Fig. 3). In BTPFs, the femoral condyles impinged both the lateral and medial plateau, leading to fracture in both locations, so the fracture locations in the lateral and medial plateau are interrelated, not isolated [15, 16]. With the extension injury mechanism, the knee joint is extended and stable at the time of injury, and rotation is negligible [17]; in this scenario, the lateral and medial impingement areas are located in the anterolateral and medial central parts of the tibial plateau [18], respectively. Under axial force, impingement results in the classic BTPF, with an anterior-to-posterior fracture line. With flexion motion, the lateral and medial femoral condyles exhibit different trajectories in the axial plane, which form flexion rotation positions, and the maximum ranges of internal and external rotation can reach 45 degrees [19], so the 
flexion mechanism can divide into two types (internal and external rotation). When the knee is injured during internal rotation and flexion, the lateral femoral condyle rolls back and externally rotates (the knee is internally rotated in relation to it) [20-22], and the lateral condyle compresses the posterolateral part of the tibial plateau, while the medial condyle moves forwards and compresses the anteromedial part of the tibial plateau $[18,22,23]$. A fracture line oriented from anteromedial to posterolateral is observed in flexioninternal rotation-type fractures. By contrast, when the injury occurs during external rotation and flexion, the femoral condyles compress the anterolateral part and posteromedial parts of the tibial plateau, respectively $[18,23]$. An anterolateral-to-posteromedial fracture line orientation is found in this type of fracture.

Posterolateral fractures are common in BTPFs, and their morphology has been described in many studies $[6,8,10,24]$; however, few studies have focused on the mechanism of injury. Currently, posterolateral fracture is believed to result from axial and valgus loading with the knee in flexion, and the femur tends to move in the posterior direction [25]. In the current study, 21 BTPFs with posterolateral compression fracture involvement resulted from this injury mechanism with the knee internally rotated. The compression morphology of posterolateral fracture is a result of the direct impingement between the lateral femoral condyle and an osteoporotic posterior lateral tibial plateau. A different posterolateral fracture morphology, the split type, was observed in 68 BTPFs; this type results from two other injury mechanisms, extension and flexion-external rotation injury mechanisms. With these two types of injury mechanisms, the lateral femoral condyle moves anteriorly and impinges on the anterolateral part of the tibial plateau; the fracture extends to the posterior part, which results in the split morphology of posterolateral fractures. This finding is consistent with the research of Zhu et al. [7], who also observed these two types of posterolateral fractures with different injury mechanisms in BTPFs and reproduced them in biomechanical experiments. However, in their research, rotational motion was not taken into consideration as a mechanistic factor.

Posteromedial fracture accounts for nearly one-quarter of the surface area of the plateau $[4,26]$, and the morphology usually belongs to the split type due to the concave geometry and bone mineral density of the medial plateau $[14,16]$. Three types of fracture lines in the medial plateau were observed in this series of BTPF cases, corresponding to the three injury mechanisms. With the extension injury mechanism, the medial femoral condyle impinges on the central part of the medial plateau [14], and the anterior-to-posterior fracture line commonly has a horizontal side branch in medial plateau. With the flexion-internal rotation injury mechanism, the femoral condyle impinges on the anterior part of the medial plateau $[14,18,19]$, resulting in an oblique fracture line (directed from anteromedial to posterolateral) and a large posteromedial fragment. These fracture characteristics have been depicted via a "fracture mapping" method by Molenaars [12], and Yang [6] reported 42 posteromedial plateau fractures with these characteristics. With the knee flexion-external rotation injury mechanism, the femoral condyle compresses the posterior part of the medial plateau $[14,18,19]$, resulting in small fragments and parallel fracture lines. Previous research has deduced that the mechanism involved in this fracture pattern is flexion and internal rotation of the medial femoral condyle (external knee rotation) $[4,27]$, which is consistent with the current findings.

Patzold et al. [28] described the difference in medial plateau fracture line orientation in 81 BTPFs and classified the fractures based on the fracture line angle (sagittal fracture line, coronal fracture line, or no 
fracture line in the medial plateau). As described by Patzold, in the axial plane, a coronal fracture line $\left(60^{\circ}-120^{\circ}\right.$ relative to the sagittal plane) in the medial plateau fracture type was accompanied by a sagittal fracture line in the lateral plateau, which is consistent with the fracture line morphology resulting from the extension injury mechanism in the current study. The sagittal fracture line type consists of two types of fracture line orientations, $0^{\circ}-30^{\circ}$ and $150^{\circ}-180^{\circ}$, which can result from flexion-internal and external rotation injury mechanisms, respectively.

The posterior fracture fragments resulting from different injury mechanisms may be fixed with different approaches and strategies by reversing the injury mechanism. For BTPFs with an extension injury mechanism, a semi-flexed position (20-40 flexion) and valgus or varus traction during the reduction procedure are recommended to counter the initial injury mechanism. The posterolateral fragment can be fixed to the anterolateral fragment through an extended anterolateral incision via a single $3.5 \mathrm{~mm}$ lateral rafting locking plate [29, 30]; a displaced posteromedial fragment always needs a posteromedial approach [3] with a buttress plate to achieve reduction [4,31]. Traction in an extended position with external or internal rotation of the tibia is useful for reducing the flexion-internal rotation and flexion-external rotation types of BTPFs, respectively. Luo et al. reported using the posteromedial approach [5, 32], retracting the medial head of the gastrocnemius to expose and fix the posterolateral and posteromedial BTPFs for flexion-internal rotation. For most of the flexion-internal rotation BTPFs, an extended anterolateral incision and a rafting plate are enough to stabilize the posterolateral fragment and a small, nondisplaced posteromedial fragment $[33,34]$.

This study has some limitations that must be considered. First, the injury mechanisms found in this study are all based on BTPFs with posterior involvement, which are the most complex fractures to understand, the most challenging to devise surgical strategies for, and always inferior in prognosis. Therefore, not all tibial plateau fractures can be explained by these mechanisms; we may further investigate other types of tibial plateau fractures in the future. Second, the findings of our research were based on 3D simulations, and one may argue that the interpretation of injury mechanisms is subjective. We believe that further biomechanical investigations will strengthen the evidence presented herein. Third, for this series of fractures caused by high-energy trauma, the ligaments were difficult to observe and reconstruct by MRI due to the serious displacement of the fragments, and so this study did not investigate the mechanisms of ligament injury.

\section{Conclusion}

In this study, we investigated the mechanisms of injury in 96 BTPFs involving the posterior part of the joint by using 3D techniques to simulate the knee position at the time of fracture; three main types and six subtypes of recurrent injury mechanisms were ultimately found. Along with these different injury mechanisms, surgeons should pay attention to the differences in fracture morphologies and the main fracture location. Additionally, given the different characteristics of the posterior fragment, the surgical approach and strategy should be chosen according to the injury mechanism. The 3D simulation method employed in this study may be a useful supplemental method for investigating the mechanisms of fracture injuries.

\section{Abbreviations}


BTPF, bicondylar tibial plateau fracture; 3D, three-dimensional; 2D, two-dimensional; CT, computed tomography; DICOM, Digital Imaging and Communications in Medicine; ANOVA, one-way analysis of variance.

\section{Declarations}

Ethics approval and consent to participate

The research is approved by the Institutional Review Board of the Third Hospital of Hebei Medical University, No. 139 Ziqiang Road, Shijiazhuang, 050051, Hebei, People's Republic of China. IRB Protocol \#2019-036-1.

Consent for publication

Not applicable

Availability of data and material

The datasets used and/or analyzed during the current study are available from the corresponding author on reasonable request.

Competing interests

The authors declare that they have no competing interests.

Funding

Not applicable

Authors' contributions

Aqin Peng designed the study collected the data and drafted the manuscript. Shuo Pan collected and analyzed the data and drafted the manuscript. Yaning Hu and Shuai Wang collected and analyzed the data. Xiao Zhang conceived the study and assisted in drafting the manuscript. All authors read and approved the final manuscript.

Acknowledgements

Not applicable

Authors' information

Not applicable

\section{References}


1 Chan PS, Klimkiewicz JJ, Luchetti WT, Esterhai JL, Kneeland JB, Dalinka MK, et al. Impact of CT scan on treatment plan and fracture classification of tibial plateau fractures. Journal of orthopaedic trauma. 1997;11(7):484-9.

2 Bhattacharyya T, McCarty LP, 3rd, Harris MB, Morrison SM, Wixted JJ, Vrahas MS, et al. The posterior shearing tibial plateau fracture: treatment and results via a posterior approach. $\mathrm{J}$ Orthop Trauma. 2005;19(5):305-10.

3 Fakler JK, Ryzewicz M, Hartshorn C, Morgan SJ, Stahel PF, Smith WR. Optimizing the management of Moore type I postero-medial split fracture dislocations of the tibial head: description of the Lobenhoffer approach. J Orthop Trauma. 2007;21(5):330-6.

4 Barei DP, O'Mara TJ, Taitsman LA, Dunbar RP, Nork SE. Frequency and fracture morphology of the posteromedial fragment in bicondylar tibial plateau fracture patterns. J Orthop Trauma. 2008;22(3):176-82.

5 Luo CF, Sun H, Zhang B, Zeng BF. Three-column fixation for complex tibial plateau fractures. J Orthop Trauma. 2010;24(11):683-92.

6 Yang G, Zhai Q, Zhu Y, Sun H, Putnis S, Luo C. The incidence of posterior tibial plateau fracture: an investigation of 525 fractures by using a CT-based classification system. Archives of orthopaedic and trauma surgery. 2013;133(7):929-34.

7 Zhu Y, Meili S, Dong M-J, Zhai Q-L, Yao L, Wang J-C, et al. Pathoanatomy and incidence of the posterolateral fractures in bicondylar tibial plateau fractures: a clinical computed tomography-based measurement and the associated biomechanical model simulation. Archives of Orthopaedic and Trauma Surgery. 2014;134(10):1369-80.

8 Sohn H-S, Yoon Y-C, Cho J-W, Cho W-T, Oh C-W, Oh J-K. Incidence and fracture morphology of posterolateral fragments in lateral and bicondylar tibial plateau fractures. Journal of orthopaedic trauma. 2015;29(2):91-7.

9 Schatzker J, McBroom R, Bruce D. The tibial plateau fracture. The Toronto experience 1968-1975. Clinical orthopaedics and related research. 1979(138):94-104.

10 Carlson DA. Posterior bicondylar tibial plateau fractures. Journal of orthopaedic trauma. 2005;19(2):73-8.

11 Armitage BM, Wijdicks CA, Tarkin IS, Schroder LK, Marek DJ, Zlowodzki M, et al. Mapping of scapular fractures with three-dimensional computed tomography. J Bone Joint Surg Am. 2009;91(9):22228.

12 Molenaars RJ, Mellema JJ, Doornberg JN, Kloen P. Tibial Plateau Fracture Characteristics: Computed Tomography Mapping of Lateral, Medial, and Bicondylar Fractures. The Journal of Bone and Joint Surgery-American Volume. 2015;97(18):1512-20. 
13 Robertson DD, Debski RE, Almusa E, Armfield DR, Stone DA, Walker PS. Knee joint biomechanics: relevance to imaging. Seminars in musculoskeletal radiology. 2003;7(1):43-58.

14 Iwaki H, Pinskerova V, Freeman MA. Tibiofemoral movement 1: the shapes and relative movements of the femur and tibia in the unloaded cadaver knee. J Bone Joint Surg Br. 2000;82(8):1189-95.

15 Martelli S, Pinskerova V. The shapes of the tibial and femoral articular surfaces in relation to tibiofemoral movement. J Bone Joint Surg Br. 2002;84(4):607-13.

16 Eggli S, Hartel MJ, Kohl S, Haupt U, Exadaktylos AK, Röder C. Unstable bicondylar tibial plateau fractures: a clinical investigation. Journal of orthopaedic trauma. 2008;22(10):673-9.

17 Kim HY, Kim KJ, Yang DS, Jeung SW, Choi HG, Choy WS. Screw-Home Movement of the Tibiofemoral Joint during Normal Gait: Three-Dimensional Analysis. Clin Orthop Surg. 2015;7(3):303-9.

18 Hill PF, Vedi V, Williams A, Iwaki H, Pinskerova V, Freeman MA. Tibiofemoral movement 2: the loaded and unloaded living knee studied by MRI. J Bone Joint Surg Br. 2000;82(8):1196-8.

19 Karrholm J, Brandsson S, Freeman MA. Tibiofemoral movement 4: changes of axial tibial rotation caused by forced rotation at the weight-bearing knee studied by RSA. J Bone Joint Surg Br. 2000;82(8):1201-3.

20 Johal P, Williams A, Wragg P, Hunt D, Gedroyc W. Tibio-femoral movement in the living knee. A study of weight bearing and non-weight bearing knee kinematics using 'interventional' MRI. J Biomech. 2005;38(2):269-76.

21 Todo S, Kadoya Y, Moilanen T, Kobayashi A, Yamano Y, Iwaki H, et al. Anteroposterior and rotational movement of femur during knee flexion. Clinical orthopaedics and related research. 1999(362):162-70.

22 Nakagawa S, Kadoya Y, Todo S, Kobayashi A, Sakamoto H, Freeman MA, et al. Tibiofemoral movement 3: full flexion in the living knee studied by MRI. J Bone Joint Surg Br. 2000;82(8):1199-200.

23 Freeman MAR, Pinskerova V. The movement of the normal tibio-femoral joint. J Biomech. 2005;38(2):197-208.

24 Chang SM, Zheng HP, Li HF, Jia YW, Huang YG, Wang X, et al. Treatment of isolated posterior coronal fracture of the lateral tibial plateau through posterolateral approach for direct exposure and buttress plate fixation. Arch Orthop Trauma Surg. 2009;129(7):955-62.

25 Zhang Y, Song L, Li X, Fang J. Flexion-valgus unicondylar tibial plateau depression fracture pattern: Classification and treatment. Injury. 2018;49(4):852-9.

26 Higgins TF, Kemper D, Klatt J. Incidence and morphology of the posteromedial fragment in bicondylar tibial plateau fractures. Journal of orthopaedic trauma. 2009;23(1):45-51. 
27 Connolly JF. The posterior shearing tibial plateau fracture: treatment and results via a posterior approach. Journal of orthopaedic trauma. 2005;19(7):508-.

28 Patzold R, Friederichs J, von Ruden C, Panzer S, Buhren V, Augat P. The pivotal role of the coronal fracture line for a new three-dimensional CT-based fracture classification of bicondylar proximal tibial fractures. Injury. 2017;48(10):2214-20.

29 Cho J-W, Samal P, Jeon Y-S, Oh C-W, Oh J-K. Rim Plating of Posterolateral Fracture Fragments (PLFs) Through a Modified Anterolateral Approach in Tibial Plateau Fractures. Journal of orthopaedic trauma. 2016;30(11):e362-e8.

30 Cho J-W, Kim J, Cho W-T, Kim J-K, Samal P, Gujjar PH, et al. Approaches and fixation of the posterolateral fracture fragment in tibial plateau fractures: a review with an emphasis on rim plating via modified anterolateral approach. International orthopaedics. 2017;41(9):1887-97.

31 Wang Y, Luo C, Zhu Y, Zhai Q, Zhan Y, Qiu W, et al. Updated Three-Column Concept in surgical treatment for tibial plateau fractures - A prospective cohort study of 287 patients. Injury. 2016;47(7):148896.

32 He X, Ye P, Hu Y, Huang L, Zhang F, Liu G, et al. A posterior inverted L-shaped approach for the treatment of posterior bicondylar tibial plateau fractures. Archives of orthopaedic and trauma surgery. 2013;133(1):23-8.

33 Cuéllar VG, Martinez D, Immerman I, Oh C, Walker PS, Egol KA. A Biomechanical Study of Posteromedial Tibial Plateau Fracture Stability: Do They All Require Fixation? Journal of orthopaedic trauma. 2015;29(7):325-30.

37 Lee AK, Cooper SA, Collinge C. Bicondylar Tibial Plateau Fractures: A Critical Analysis Review. JBJS Rev. 2018;6(2):e4-e.

\section{Tables}

TABLE I Baseline Demographic 


\begin{tabular}{cccc} 
Patients Age (years) & Gender & $\begin{array}{c}\text { Fracture } \\
\text { side }\end{array}$ & $\begin{array}{c}\text { Schatzker } \\
\text { classification }\end{array}$ \\
\cline { 2 - 4 } & MaleFemale Left Right & Type V & Type VI
\end{tabular}

\section{Extension}

$\begin{array}{ccccccccc}\text { Valgus } & 44 & 47.14 \pm 9.94 & 28 & 16 & 24 & 20 & 3 & 41 \\ \text { Varus } & 16 & 39.94 \pm 12.95 & 14 & 2 & 8 & 8 & 2 & 14\end{array}$

Flexion-Internal rotation

$\begin{array}{ccccccccc}\text { Valgus } & 12 & 41.75 \pm 12.77 & 7 & 5 & 6 & 6 & 2 & 10 \\ \text { Varus } & 9 & 43.89 \pm 8.13 & 8 & 1 & 5 & 4 & 2 & 7\end{array}$

Flexion-External rotation

\begin{tabular}{lccccccccc} 
& Valgus & 12 & $43.76 \pm 10.45$ & 10 & 2 & 4 & 8 & 3 & 9 \\
& Varus & 3 & $43 \pm 15.87$ & 3 & 0 & 2 & 1 & 1 & 2 \\
Total & 96 & $44.33 \pm 11.03$ & 70 & 26 & 49 & 47 & 13 & 83 \\
& & & & & & & & \\
P value & & $0.286^{\mathrm{a}}$ & $0.211^{\mathrm{b}}$ & $0.847^{\mathrm{b}}$ & \multicolumn{2}{c}{$0.227^{\mathrm{b}}$} \\
\hline
\end{tabular}

a one-way analysis of variance, ANOVA $₫$ b Fisher's Exact Test.

TABLE II Fracture Characteristics and Knee Position of Different Injury Mechanism Types

\begin{tabular}{|c|c|c|c|c|c|}
\hline \multirow{2}{*}{\multicolumn{3}{|c|}{ Knee position }} & \multicolumn{3}{|c|}{ Fracture characteristic } \\
\hline & & & $\begin{array}{l}\text { Lateral plateau fracture } \\
\text { morphology }\end{array}$ & $\begin{array}{r}\text { Medial platea } \\
\text { line orien }\end{array}$ & $\begin{array}{l}\text { i fracture } \\
\text { ation }\end{array}$ \\
\hline$\overline{\text { Sagittal }}$ & Axial & oronal & $\begin{array}{c}\text { Anterolateral Posterolatera } \\
\text { plateau plateau }\end{array}$ & $\begin{array}{l}\text { Main fracture } \\
\text { line }\end{array}$ & $\begin{array}{l}\text { Sub- } \\
\text { fracture } \\
\text { line }\end{array}$ \\
\hline & $\begin{array}{c}-10^{\circ} \\
\text { to } \\
10^{\circ}\end{array}$ & $\begin{array}{l}<0^{\circ} \\
>0^{\circ}\end{array}$ & ress & $\begin{array}{l}\text { anterior to } \\
\text { posterior }\end{array}$ & ans \\
\hline
\end{tabular}

Flexion-

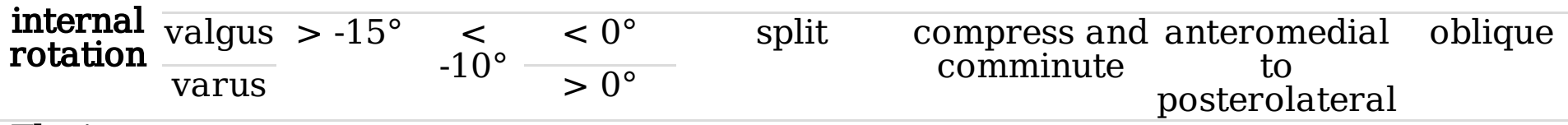

Flexion-

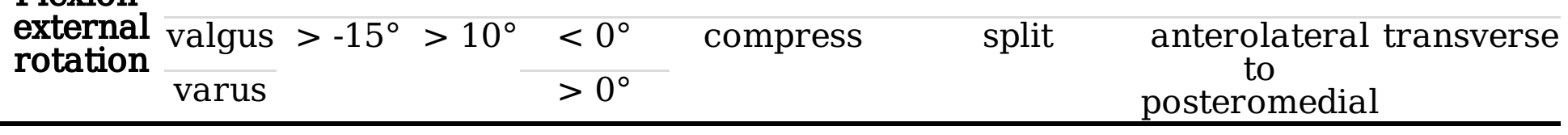


TABLE III Knee Position Data Analysis

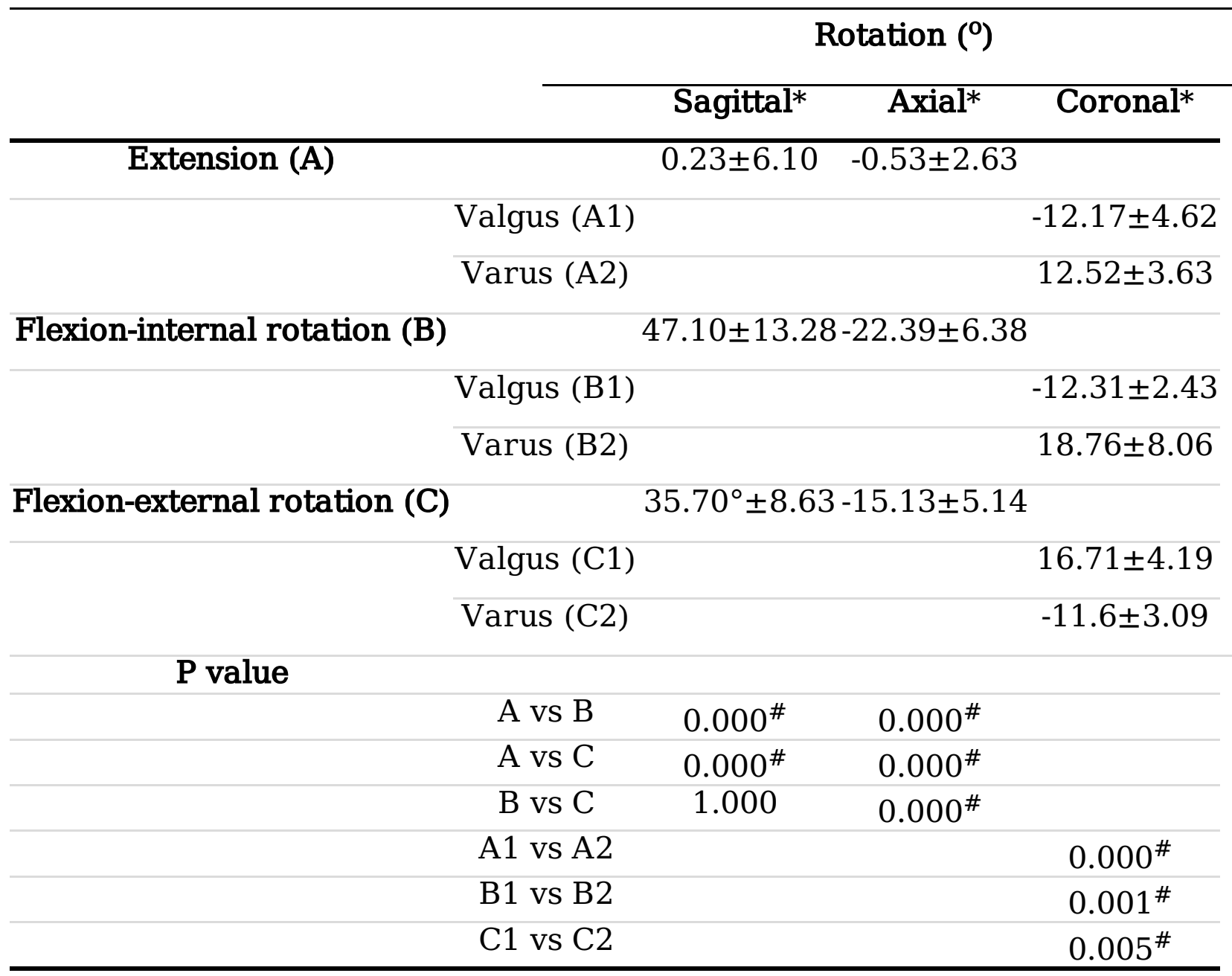

*The values are given as the mean and standard deviation. Kruskal-wallis Test was applied for all groups comparison and Nemenyi Test for comparison between the individual groups. \# The difference is significant.

\section{Figures}



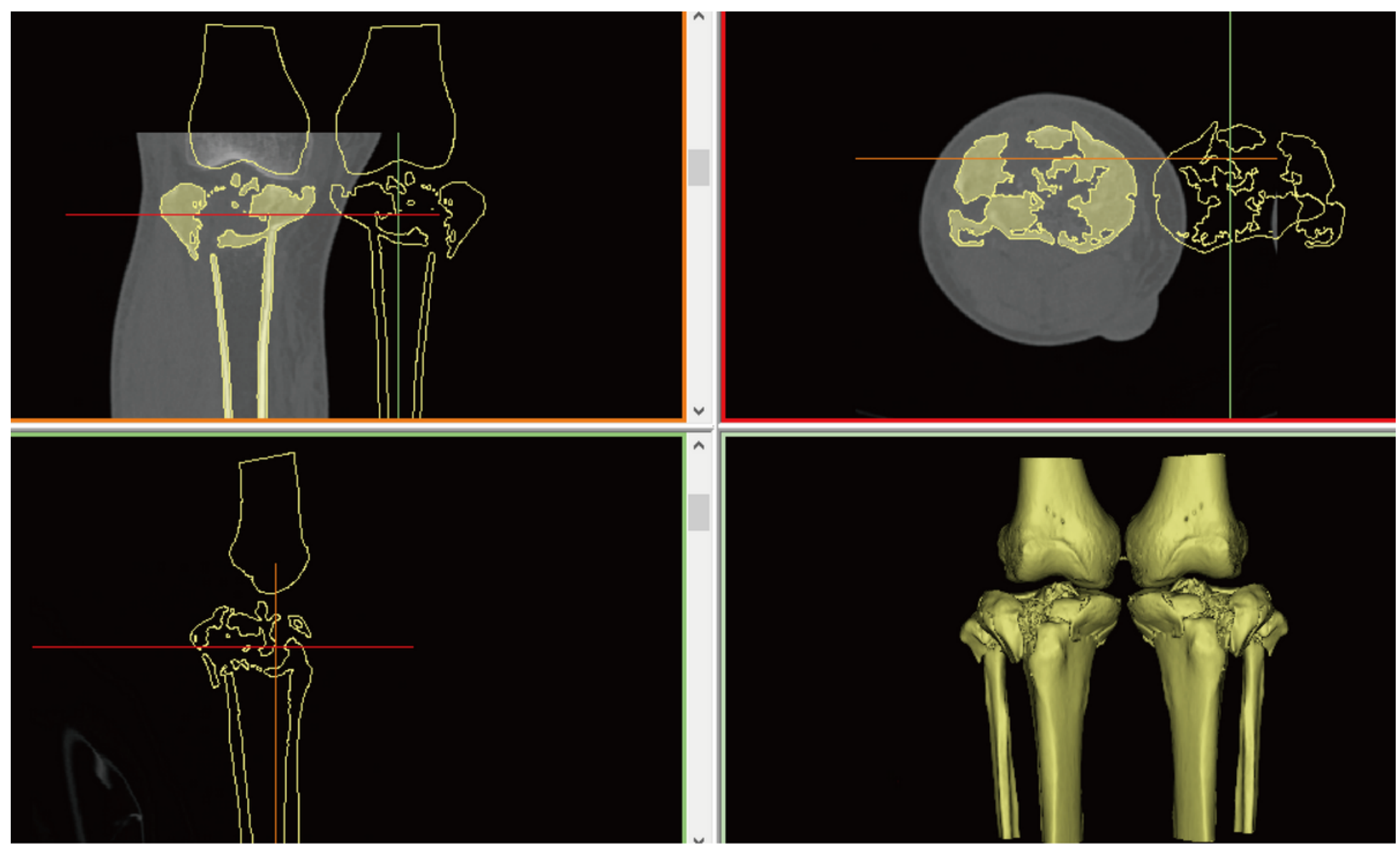

\section{Figure 1}

A right BTPF was flipped and adjusted to obtain a standard left knee model in Mimics software. 

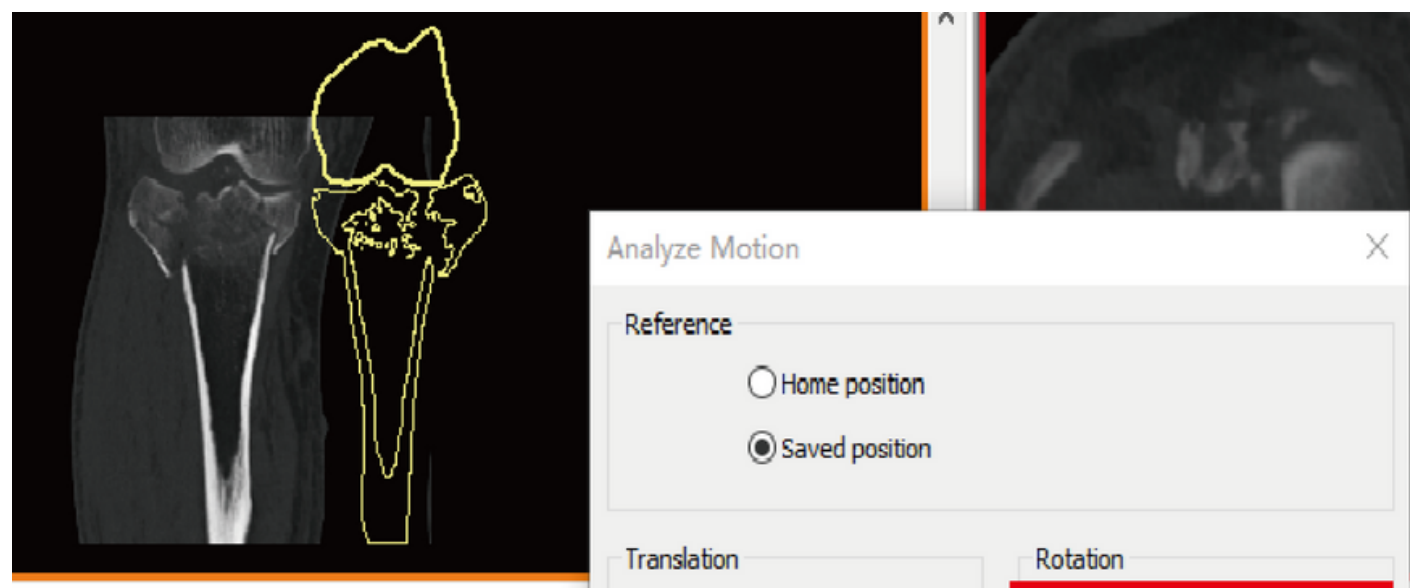

Analyze Motion

Reference

OHome position

C) Saved position
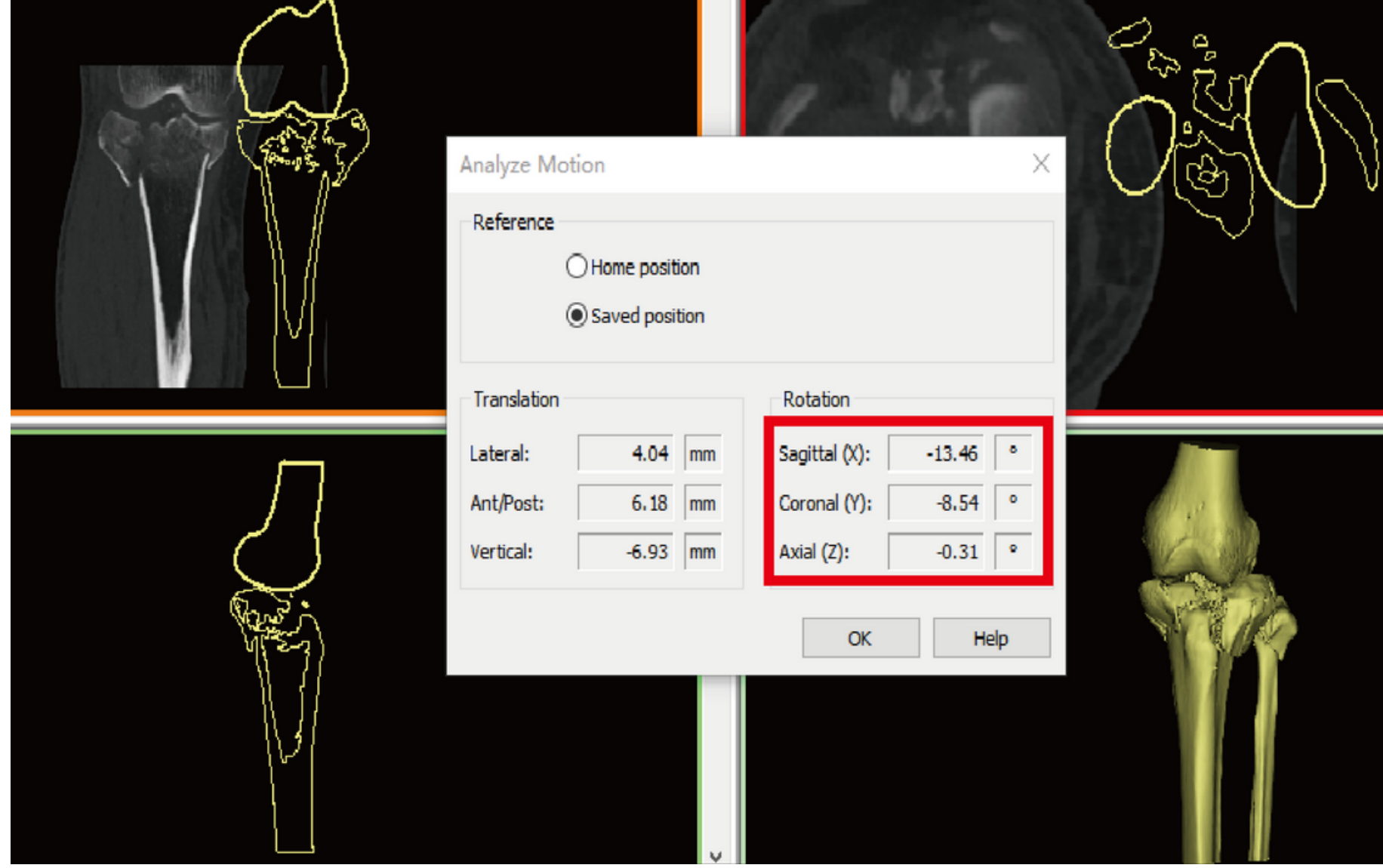

OK

Help

\section{Figure 2}

The knee position simulation and analysis. Move the femur to matched the articular surfaces of BTPF. The femoral motion in the three planes were analysed by the software. 


\section{A}

\section{Extension}
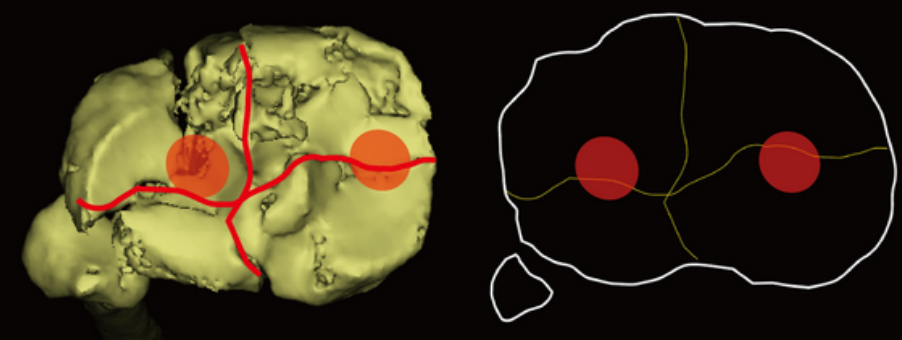

\section{Flexion \\ Internal \\ rotation}
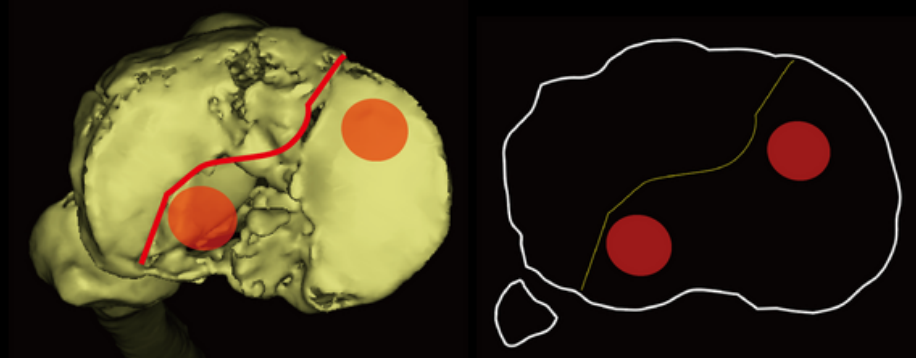

\section{Flexion External rotation}
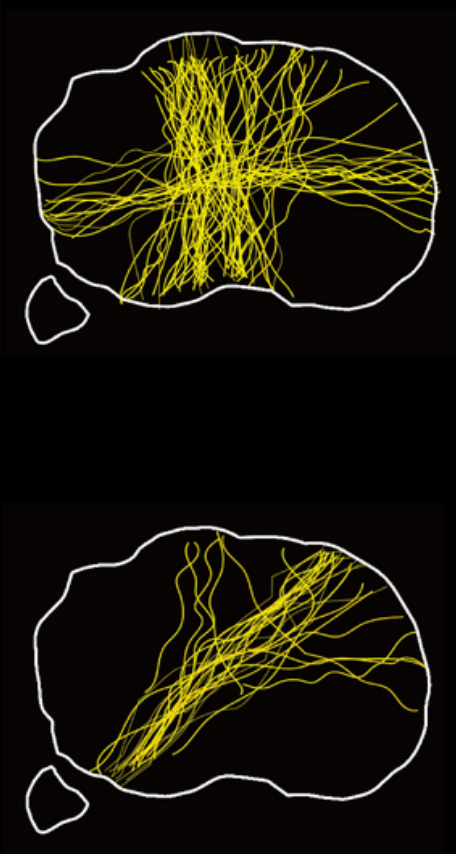

B

C

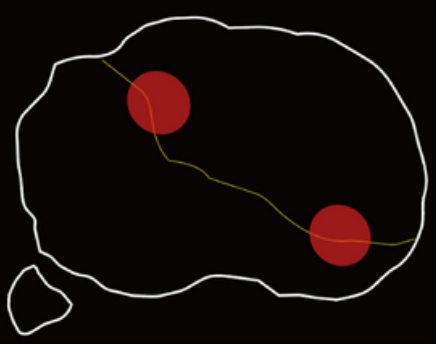

\section{Figure 3}

The impingement areas and fracture line in the three main injury mechanism types. The top view images of the 3D tibial models (Fig. 3-A column) were imported on a template. The main fracture line depicted in the template (Fig. 3-B column). Fracture lines mapping in Fig.3-C column. 


\section{Extension type}

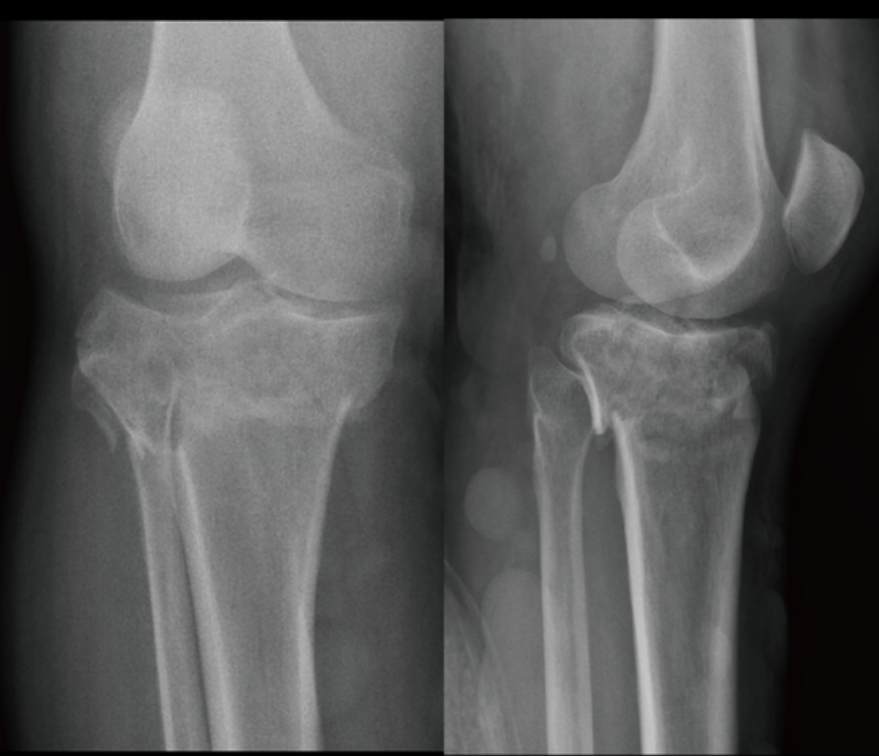

\section{Pos}

fragment

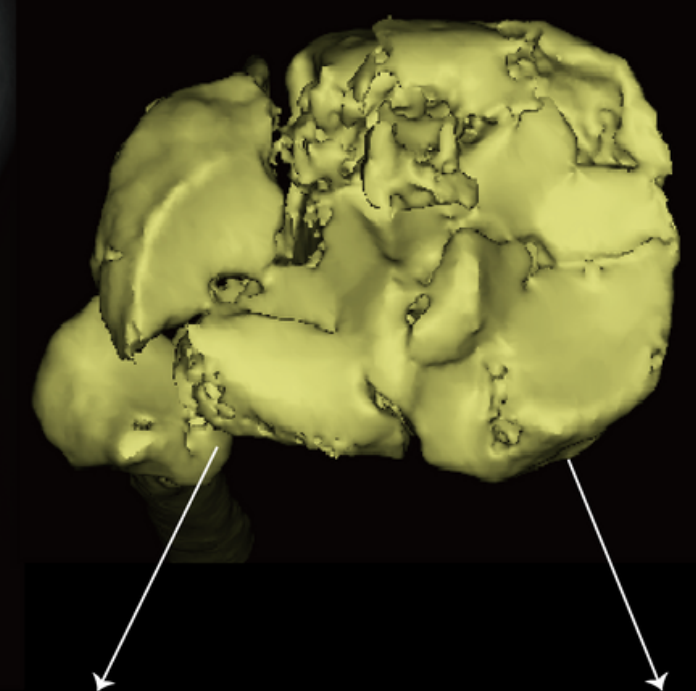

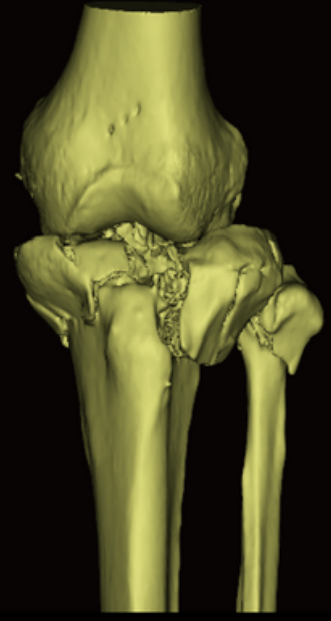

Front

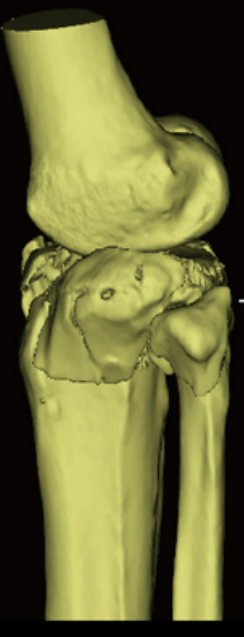

Lateral

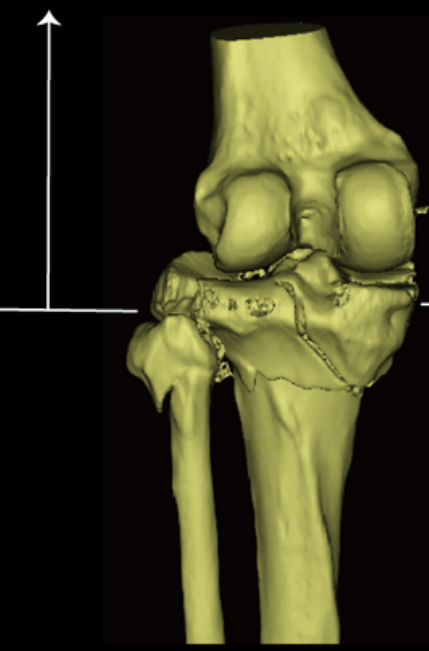

Back
Posteromedial fragment

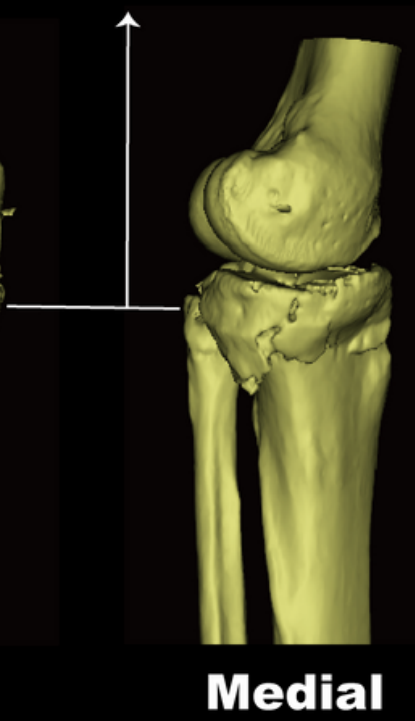

\section{Figure 4}

Fracture characteristics of BTPFs involving the posterior fragment in extension injury mechanism type. 


\section{Flexion Internal rotation type}
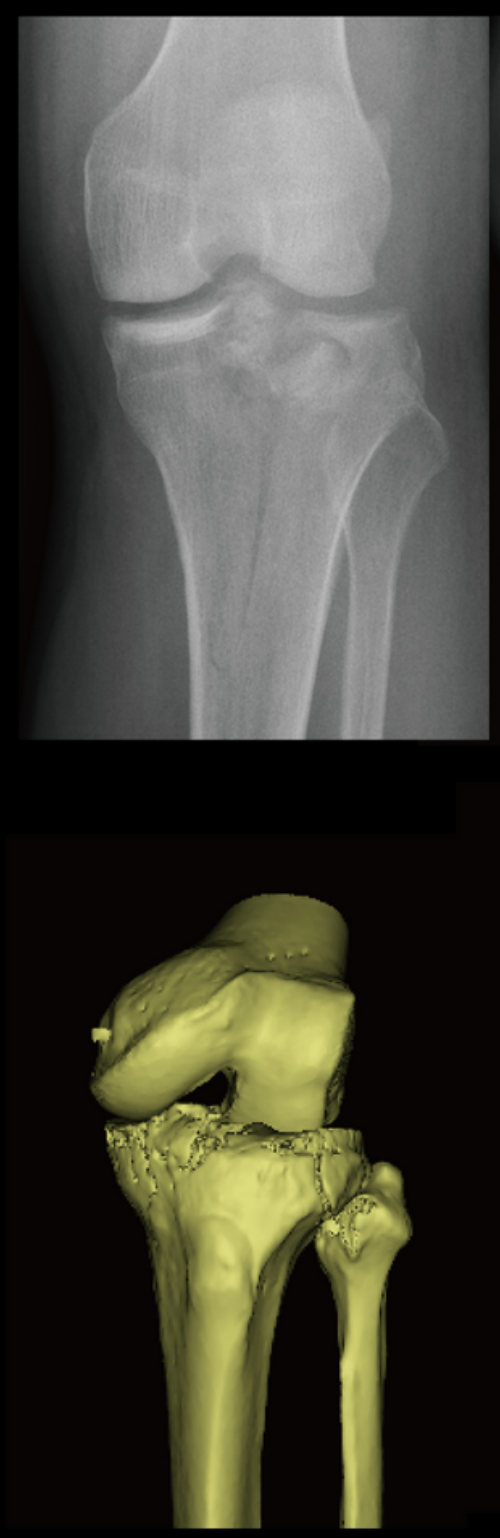

Front

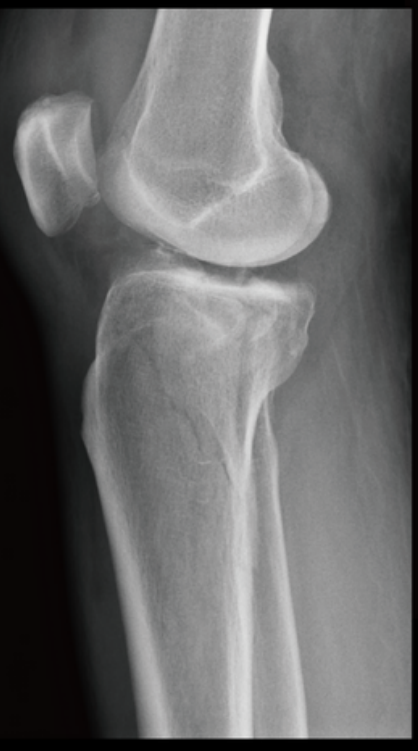

Posterolateral fragment

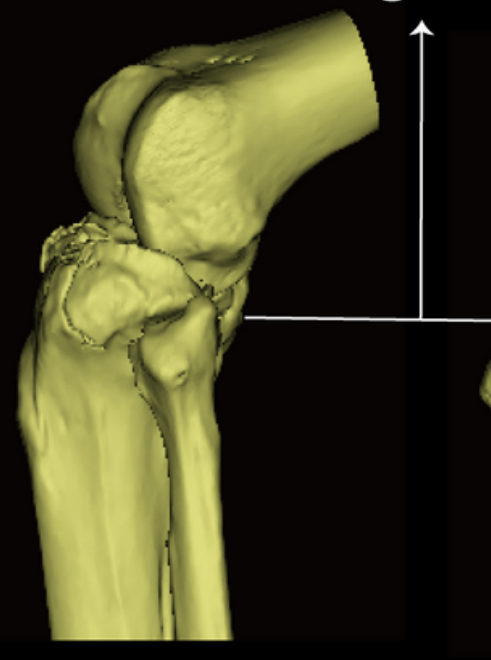

Lateral

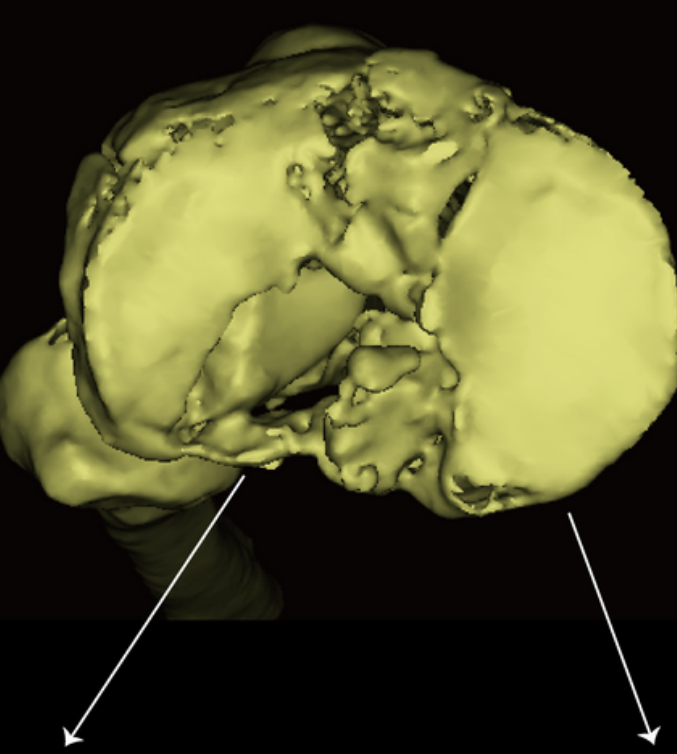

Posteromedial fragment

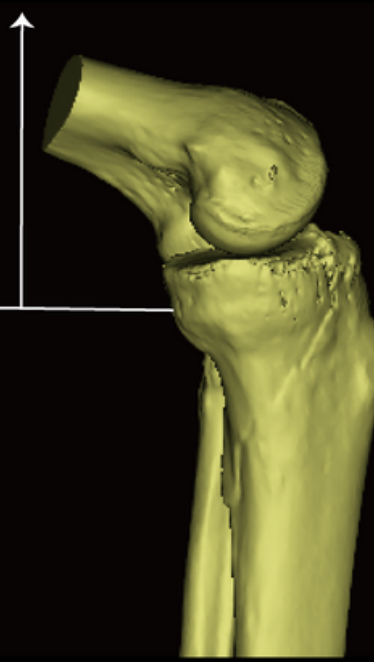

Back

\section{Figure 5}

Fracture characteristics of BTPFs involving the posterior fragment in flexion-internal rotation injury mechanism type. 


\section{Flexion External rotation type}

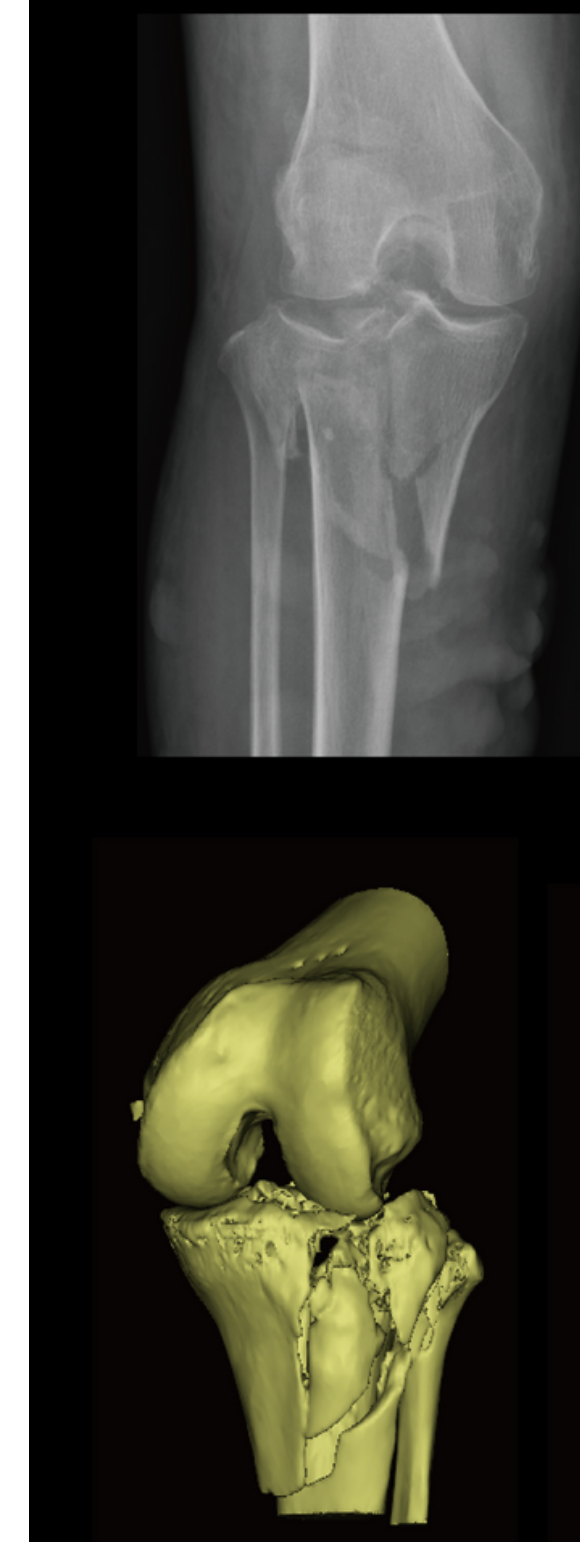

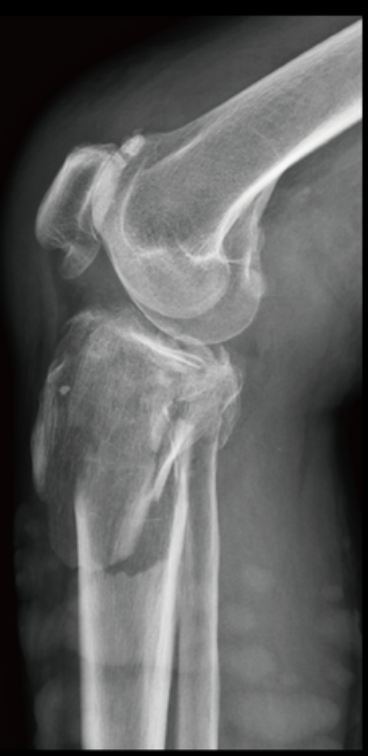

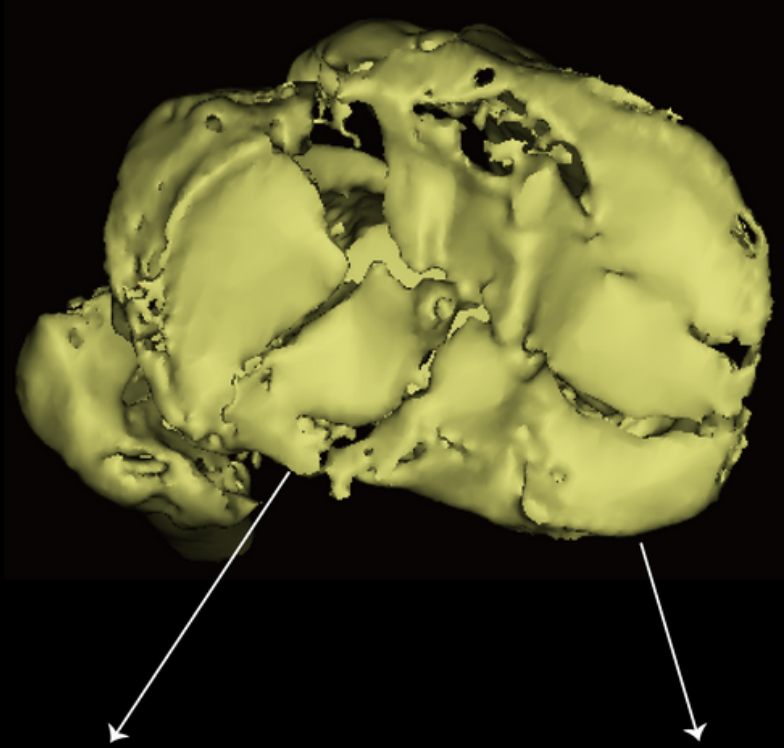

Posteromedial fragment
Posterolateral fragment

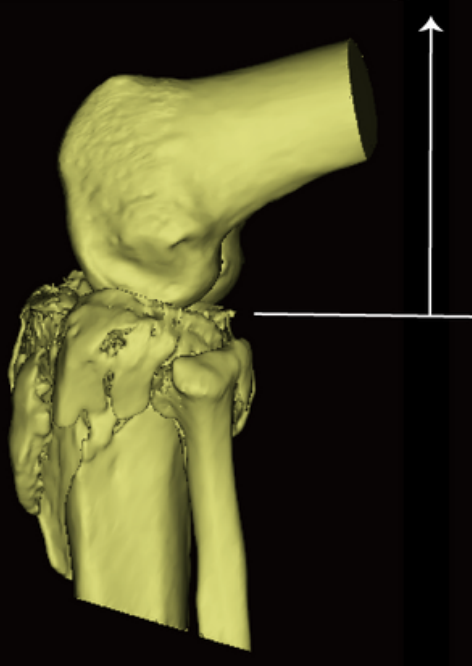

Lateral
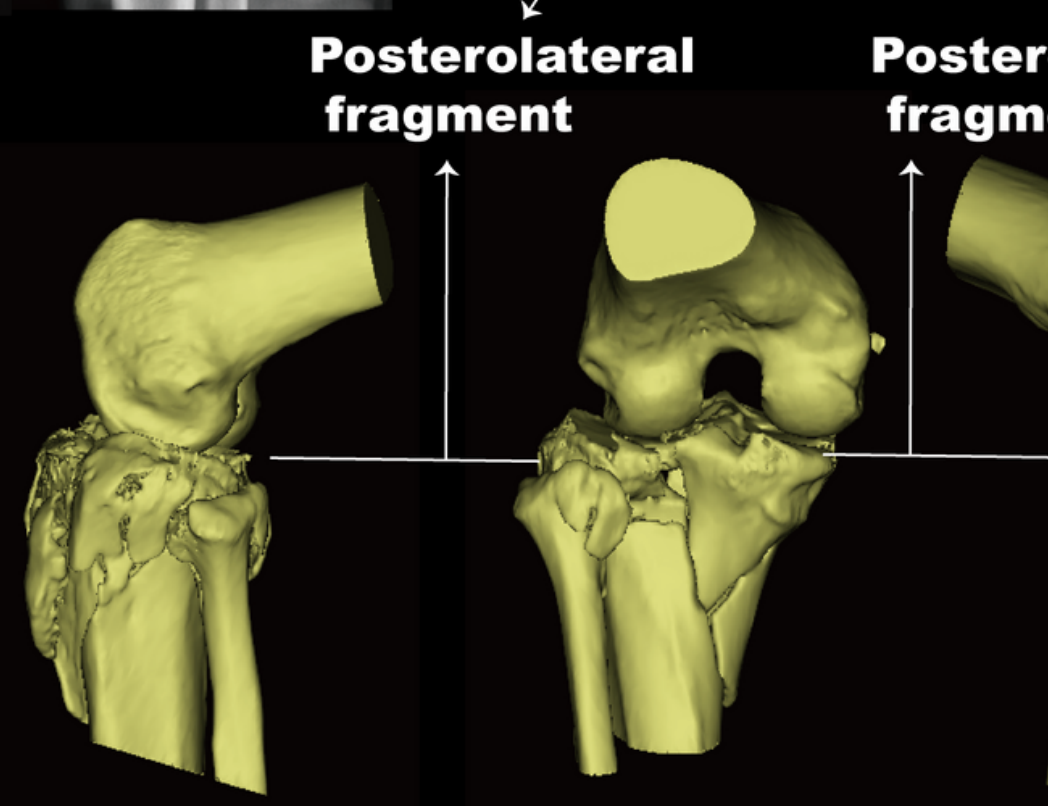

Back
Front

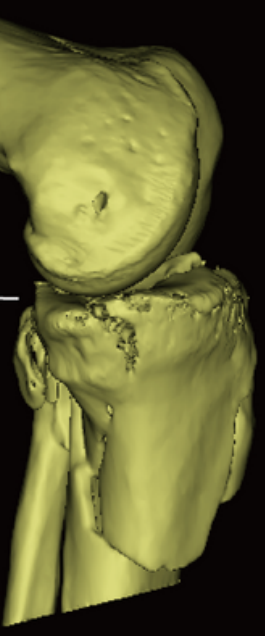

Medial

\section{Figure 6}

Fracture characteristics of BTPFs involving the posterior fragment in flexion-external rotation injury mechanism type. 